\title{
A Meta-Ensemble Classifier Approach: Random Rotation Forest
}

\author{
E. TAŞCI
}

\begin{abstract}
Ensemble learning is a popular and intensively studied field in machine learning and pattern recognition to increase the performance of the classification. Random forest is very important for giving fast and effective results. On the other hand, Rotation Forest can get better performance than Random Forest. In this study, we present a meta-ensemble classifier, called Random Rotation Forest to utilize and combine the advantages of two classifiers (e.g. Rotation Forest and Random Forest). In the experimental studies, we use three base learners (namely, J48, REPTree, and Random Forest) and two metalearners (namely, Bagging and Rotation Forest) for ensemble classification on five datasets in UCI Machine Learning Repository. The experimental results indicate that Random Rotation Forest gives promising results according to base learners and bagging ensemble approaches in terms of accuracy rates, AUC, precision, recall, and F-measure values. Our method can be used for image/pattern recognition and machine learning problems.
\end{abstract}

Index Terms - Ensemble learning, Machine learning, Pattern recognition, Data mining, Classification, Rotation forest.

\section{INTRODUCTION}

$\mathrm{E}$ NSEMBLE SYSTEMS, MULTIPLE CLASSIFIER

systems aim to combine individual decisions of a set of classifiers in some way typically by weighted or unweighted voting to classify new examples as an active research area of machine learning [1]. These systems specifically focus on obtaining a better classification performance than using a single model.

In recent years, ensemble learning has been extensively employed in a variety of subjects such as image classification, pattern recognition, remote sensing, text mining for its outstanding results [2-5]. Ensemble methods are composed of many base (weak) learners to provide data and model diversity. Types of decision tree such as CART [6], J48, REPTree [7] are commonly utilized as a base learner.

Random Forest, introduced by Breiman [8], is an extension of a bagging method and is a competitor to boosting such as AdaBoost. Thanks to its relatively fast and highly accurate

ERDDAL TASCI, is with Department of Computer Engineering, Ege University, Izmir, Turkey, (e-mail: arif.erdal.tasci@ege.edu.tr).

(iD https://orcid.org/0000-0001-6754-2187

Manuscript received December 25, 2018; accepted April 2, 2019. DOI: $10.17694 /$ bajece. 502156 prediction, measures of variable importance, it is very attractive for classification problems. Random Forest can also deal with missing values.

On the other hand, Rotation Forest, proposed by Rodriguez et al. [9], generates classifier ensembles based on feature extraction. This ensemble learning method produces more accurate results than AdaBoost and Random Forest. It constructs individual classifiers more diverse than Bagging [9].

When both ensemble methods are compared, rotation forest applies Principal Component Analysis (PCA) on randomly selected feature subsets to reconstruct full feature space, promote the diversity and increase the accuracy of classifiers. In random forest, random selection of features to split each node provides error rates that compare favorably to AdaBoost [10].

The main contribution of this paper is summarized as follows: i) A meta-ensemble method which uses Random Forest as a base learner in the Rotation Forest ii) A comparative analysis of three base learners and two meta ensemble learners on five datasets in UCI Machine Learning Repository in terms of five evaluation criteria: accuracy, weighted area under curve (AUC), precision, recall and Fmeasure.

The remainder of this paper is organized as follows: In Section II, related works specific to rotation forest are presented. In Section III, the proposed method is described in detail. Definition of the datasets, experimental process and results are given in Section IV. Finally, Section V mentions the conclusion and direction of future works.

\section{RELATED WORK}

Classifications based on rotation forest method are commonly used in various fields such as machine learning, image recognition. Researchers developed improved versions of this ensemble method in their studies.

In [2], a new weight-based rotation forest (WRoF) ensemble algorithm is proposed for the classification of the hyperspectral image. Experimental results on two real hyperspectral datasets demonstrate that the WRoF algorithm results in significant improvement compared with random forests and rotation forest.

In [3], GA feature selection and Rotation Forest are used for Breast cancer diagnosis. Feature selection stage aims to reduce the computational complexity and speed up the data mining process. Their proposed approach yields the enhancement in performances. 
In [11], PCA, non-parametric discriminant analysis (NDA), random projections (RP) and independent component analysis (ICA) are applied to feature transformation in the original Rotation Forest for microarray dataset based cancer classification. According to the results, ICA improves the performance of Rotation Forest compared with the other transformation methods.

In [12], an ensemble classifier, called RotBoost, is proposed by combining Rotation Forest and AdaBoost. They used 36 real-world data sets from the UCI repository, among which a classification tree is adopted as the base learning algorithm. Their results show that RotBoost can generate ensemble classifiers with significantly lower prediction error than either Rotation Forest or AdaBoost.

In [13], firstly correlation-based feature selection (CFS) algorithm is performed for feature reduction. Then, ensemble methods are applied to medical datasets to increase classification accuracy.

In [14], Random Forest and Rotation Forest are applied to fully polarized SAR image classification using polarimetric and spatial features. Then, they conclude that Rotation Forest can get better accuracy than SVM and Random Forest, whereas Random Forest is much faster than Rotation Forest.

In [15], a classification method, called improved Rotation Forest (ROF) is proposed. In this method, Non-negative matrix factorization (NMF) is used to do feature segmentation to get more effective data. Then, kernel extreme learning machine (KELM) is chosen as a base classifier. Q-statistic is used to select base classifiers.

In [16], rotation forest is investigated for problems with continuous features whether this method is the best classifier or not.

\section{Methodology}

This section presents the overview of the proposed system and describes data preprocessing stage, classification algorithms, ensemble learning methods and the algorithmic complexity of the method used in this study in detail.

\section{A. Overview of the Proposed System}

Overview of the proposed system is shown in Fig. 1. This system consists of several stages: datasets, base, and metalearners, comparative analysis of results, conclusion and future work. In addition, 10-fold cross validation used for all learners and datasets to obtain generalization performance of the system is shown.

\section{B. Data Preprocessing}

In this stage, the ranges of the values of the data from diverse machine learning datasets may be high. In this case, some features can affect classification algorithms significantly or negatively. Therefore, data values are normalized to $[0,1]$ range using min-max normalization technique [17]. For mapping a value, of a feature $x_{i}$ from the range $\left[\min \left(x_{i}\right)\right.$, $\left.\max \left(\mathrm{x}_{\mathrm{i}}\right)\right]$ to a new range $\left[\operatorname{minx}_{\text {new }}, \max _{\text {new }}\right]$, the normalized feature $\hat{\mathrm{x}}_{\mathrm{i}}$ is computed as Eq. 1 .

$\hat{x}_{i}=\frac{x_{i}-\min _{x_{i}}}{\max _{x_{i}}-\min _{x_{i}}} \cdot\left(\max x_{\text {new }}-\min x_{\text {new }}\right)+\min x_{\text {new }}$

\section{Classification Algorithms}

In this study, three base learners including J48, REPTree, and Random Forest are employed.

The J48 classifier is the extension of decision tree ID3 and straightforward C4.5 algorithms with additional features like accounting for missing values, continuous attribute value, and derivation of rules $[18,19]$. This classifier utilizes top-down and greedy search through all possible branches to construct a decision tree [19].

REPTree is a fast decision tree classifier algorithm. It constructs the decision tree using entropy and information gain of the attribute with reduced error pruning technique [18]. It builds multiple trees and selects the best tree from the generated list of trees [18]. This algorithm prunes the tree with the back fitting method.

The Random Forest classifier includes a set of tree classifiers. Each classifier is generated independently using randomly selected subspaces of data. Then, each tree assigns a unit vote for the most popular class to classify an input pattern with majority voting. Tree randomly samples the attributes, chooses and calculates the best split among those variables instead of the best split among all attributes in the training set [14]. The tree is grown using CART methodology to maximum size, without pruning [20].

Random Forest is considered to be one of the most accurate general-purpose learning techniques [20]. This algorithm is simple, easily parallelized, robust to outliers and noise, faster than bagging and boosting [8].

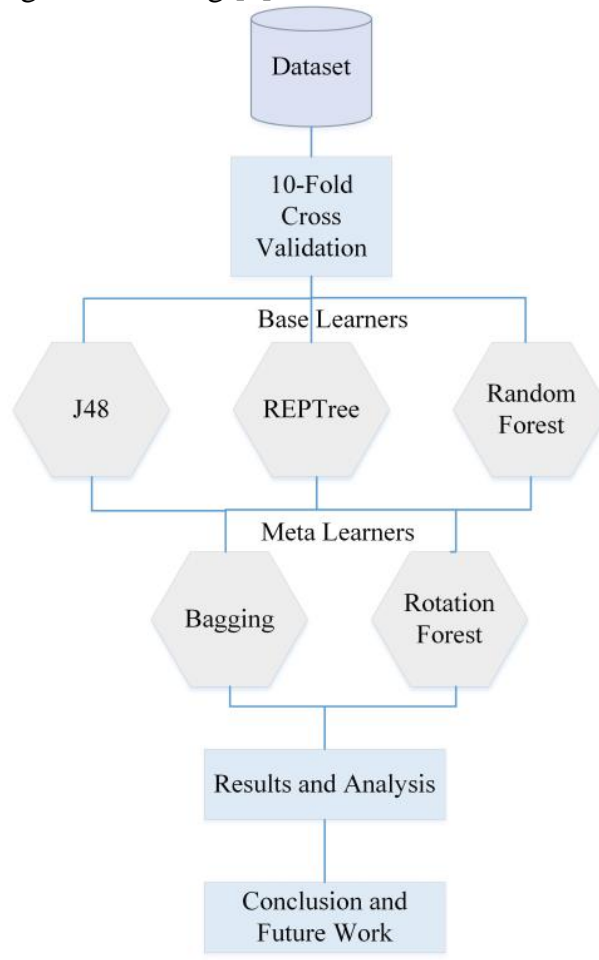

Fig.1. Overview of the proposed system

\section{Ensemble Learning Methods}

To evaluate the predictive performance, two ensemble algorithms consisting of Bagging and Rotation Forest are utilized. In addition, we introduce a forest-in-forest or meta 
ensemble learning method (e.g. Random Rotation Forest) for effective classification results.

Bagging (short for bootstrap aggregating) algorithm is one of the earliest, simple, effective and popular ensemble learning approach. This algorithm generates an ensemble of independent models in which each base learner is trained using a sample of instances taken from the original dataset with replacement [21]. Bagging can be easily parallelized due to the fact that base learners are independently trained. It is best suited for problems with relatively small available training datasets [22].

Rotation Forest is an ensemble learning method that trains base learners on the whole dataset in a rotated feature space [21]. In this method, the features are randomly split into $\mathrm{K}$ subsets and PCA is applied to each subset for each base classifier in the ensemble [9]. The average confidence for each class and all classifiers are calculated. Then, the final class label is determined to the one with the highest confidence value [14].

\section{E. The Algorithmic Complexity of the Proposed Method}

The time complexity of Random Forest depends on the number of randomized trees $(\mathrm{M})$, samples $(\mathrm{N})$, and variables (p) randomly drawn at each node. Accordingly, the time complexity for building Random Forest is $\mathrm{O}\left(\mathrm{MKN} \tilde{N}^{2} \log \tilde{N}\right)$ [23]. $\tilde{\mathrm{N}}=0.632 \mathrm{~N}$, owing to the fact that bootstrap samples draw, on average, $63.2 \%$ of unique samples [23].

Similar to Random Forest, the time complexity of Rotation Forest depends on the number of PCA (P) for randomly splitting feature subsets, the number of iterations (I) and base learners (B) [9]. For the proposed method, the time complexity of Random Rotation Forest can be calculated as $\mathrm{O}\left(\mathrm{PIMKN} \tilde{N}^{2} \log \tilde{N}\right)$ due to the fact that we use Random Forest as a base learner.

The memory requirement of the proposed algorithm is directly proportional to the number of iterations (I), the number of trees (T), selected feature counts (F) and the size of the dataset $(\mathrm{S})$. So, the proposed algorithm can use the space of $\left(\mathrm{I}^{*} \mathrm{~T}^{*} \mathrm{~F}^{*} \mathrm{~S}\right)$.

\section{EXPERIMENTAL DESIGN}

We describe and present the experimental process, evaluation measures and experimental results for this study in the subsections.

\section{A. Experimental Process}

In this study, five datasets from UCI Machine Learning Repository [24] were utilized for classification schemes. These datasets are commonly used for machine learning problems. They also have numerical attributes and no missing values. The characteristics of these datasets are illustrated in Table I.

TABLE I

CHARACTERISTICS OF THE DATASETS USED IN THIS STUDY

\begin{tabular}{|l|c|c|c|}
\hline Dataset & Size & Features & Classes \\
\hline diabetes & 768 & 8 & 2 \\
\hline heart-statlog & 270 & 13 & 2 \\
\hline ionosphere & 351 & 34 & 2 \\
\hline messidor_features & 1151 & 19 & 2 \\
\hline wifi & 2000 & 7 & 4 \\
\hline
\end{tabular}

In all experiments, J48, REPTree and Random Forest are chosen as base classifiers. In addition, bagging and rotation forest are used as meta-learners. All experiments are performed on total 9 schemes including base and ensemble learners by using WEKA (Waikato Environment for Knowledge Analysis) machine learning toolkit and JAVA programming language. We utilized default parameter values for all classifiers in WEKA.

We carry out a 10 -fold cross validation to all datasets to yield reliable results for unseen data. For 10-fold crossvalidation, the original dataset is randomly partitioned into 10 equally sized sets, one of which is used as a validation for testing, while the remaining sets are used for training operations. The process is repeated 10 times and calculated the averages of the results.

\section{B. Evaluation Measures}

To evaluate the performance of the proposed method, we employed 5 different evaluation measures including accuracy rate, weighted AUC, weighted precision, weighted recall, and weighted F-measure.

Classification accuracy (ACC) is represented as the proportion of the total number of true positives and true negatives over the total number of instances. The equation of accuracy rate is shown in Eq. 2.

$$
\mathrm{ACC}=\frac{\mathrm{TP}+\mathrm{TN}}{\mathrm{TP}+\mathrm{TN}+\mathrm{FP}+\mathrm{FN}}
$$

where TP, TN, FP, and FN denote the number of True Positives, True Negatives, False Positives, and False Negatives, respectively.

AUC is the area under the ROC curve for classifier performance. Its value will always be between 0.0 and 1.0 . ROC graphs are two-dimensional graphs. In this curve, TP rate is plotted on the $\mathrm{Y}$-axis and FP rate is plotted on the $\mathrm{X}$ axis [25]. If AUC value is close to 1, the classifier is stronger and better than random classifier.

Precision is the positive predictive value [25]. It is the ratio of the number of true positives to the total number of true positives and false positives. The equation is illustrated in Eq. 3.

$$
\text { Precision }=\frac{\mathrm{TP}}{\mathrm{TP}+\mathrm{FP}}
$$

Recall is the true positive rate or hit rate [25]. It is the ratio of the number of true positives to the total number of true positives and false negatives (e.g. total number of positives). The equation is illustrated in Eq. 4.

$$
\text { Recall }=\frac{\mathrm{TP}}{\mathrm{TP}+\mathrm{FN}}
$$

F-measure is defined in Eq. 5 [25]. This measure depends on precision and recall values.

$$
\mathrm{F}-\text { measure }=\frac{2}{1 / \text { precision }+1 / \text { recall }}
$$

In the weighting operation, these criteria are adjusted by the reference class's prevalence proportionally in the data [26]. 


\section{Experimental Results}

Table II presents classification accuracies for all datasets, base and ensemble learners. As it can be observed from Table II, Random Rotation Forest gives highly accurate results than other approaches except for heart-statlog dataset. In addition to the fact that Random forest produces more accurate results than J48 and REPTree, Rotation Forest is suitable for metalearner.

In Table III, weighted AUC values are introduced for all datasets, base and ensemble classifiers. According to Table III, Random Rotation Forest gives the best results very close or equal to 1.0. So, it can be concluded that Random Rotation Forest is very strong and effective classifier for machine learning tasks used in this study.
In Table IV, weighted precision values obtained by all base and ensemble classifiers for all datasets are presented. As it can be observed from Table IV, Random Rotation Forest produces similar results to Table II. When this meta ensemble learning method is used, the performance of the system increases significantly.

In Table V and VI, weighted recall and weighted F-measure values are illustrated for all datasets, base and ensemble classifiers, respectively. These values give similar results compared to Table II and IV.

As well as these given tables, to show the effects of the classifiers and datasets, the charts are given in Fig. 2-6.

TABLE II

CLASSIFICATION ACCURACIES (\%) FOR UCI DATASETS

\begin{tabular}{|l|c|c|c|c|c|c|c|c|c|}
\cline { 2 - 11 } & \multicolumn{3}{c}{ Base Learner } & \multicolumn{4}{c|}{$\begin{array}{c}\text { Meta Learner } \\
\text { Bagging }\end{array}$} & \multicolumn{3}{c|}{$\begin{array}{c}\text { Meta Learner } \\
\text { Rotation Forest }\end{array}$} \\
\hline Datasets & J48 & REPTree & Random Forest & J48 & REPTree & Random Forest & J48 & REPTree & Random Forest \\
\hline diabetes & 72.01 & 73.70 & 75.78 & 74.22 & 75.78 & 76.43 & 75.78 & 76.95 & $\mathbf{7 7 . 4 7}$ \\
\hline heart-statlog & 79.26 & 76.30 & $\mathbf{8 3 . 3 3}$ & 79.63 & 81.48 & 82.59 & 80.37 & 81.11 & 81.85 \\
\hline ionosphere & 90.31 & 89.74 & 93.73 & 92.31 & 90.88 & 93.73 & 93.45 & 93.16 & $\mathbf{9 4 . 8 7}$ \\
\hline messidor_features & 65.33 & 63.42 & 69.42 & 66.38 & 66.81 & 68.20 & 68.72 & 72.81 & $\mathbf{7 3 . 7 6}$ \\
\hline wifi & 97.40 & 97.20 & 98.30 & 97.65 & 97.10 & 98.45 & 98.40 & 98.00 & $\mathbf{9 8 . 5 5}$ \\
\hline
\end{tabular}

TABLE III

WEIGHTED AUC VALUES FOR UCI DATASETS

\begin{tabular}{|l|c|c|c|c|c|c|c|c|c|}
\cline { 2 - 11 } \multicolumn{1}{c|}{} & \multicolumn{4}{c|}{ Base Learner } & \multicolumn{4}{c|}{$\begin{array}{c}\text { Meta Learner } \\
\text { Bagging }\end{array}$} & \multicolumn{3}{c|}{$\begin{array}{c}\text { Meta Learner } \\
\text { Rotation Forest }\end{array}$} \\
\hline Datasets & J48 & REPTree & Random Forest & J48 & REPTree & Random Forest & J48 & REPTree & Random Forest \\
\hline diabetes & 0.74 & 0.78 & 0.83 & 0.82 & 0.83 & 0.83 & 0.82 & 0.83 & $\mathbf{0 . 8 3}$ \\
\hline heart-statlog & 0.77 & 0.80 & 0.90 & 0.88 & 0.87 & 0.90 & 0.88 & 0.88 & $\mathbf{0 . 9 0}$ \\
\hline ionosphere & 0.90 & 0.90 & 0.98 & 0.95 & 0.94 & 0.98 & 0.97 & 0.97 & $\mathbf{0 . 9 9}$ \\
\hline messidor_features & 0.68 & 0.66 & 0.76 & 0.74 & 0.73 & 0.76 & 0.74 & 0.81 & $\mathbf{0 . 8 2}$ \\
\hline wifi & 0.99 & 0.99 & 1.00 & 1.00 & 1.00 & 1.00 & 1.00 & 1.00 & $\mathbf{1 . 0 0}$ \\
\hline
\end{tabular}

TABLE IV

WEIGHTED PRECISION VALUES FOR UCI DATASETS

\begin{tabular}{|l|c|c|c|c|c|c|c|c|c|}
\cline { 2 - 11 } & \multicolumn{4}{|c|}{ Base Learner } & \multicolumn{3}{c|}{$\begin{array}{c}\text { Meta Learner } \\
\text { Bagging }\end{array}$} & \multicolumn{3}{c|}{$\begin{array}{c}\text { Meta Learner } \\
\text { Rotation Forest }\end{array}$} \\
\hline Datasets & J48 & REPTree & Random Forest & J48 & REPTree & Random Forest & J48 & REPTree & Random Forest \\
\hline diabetes & 0.72 & 0.73 & 0.75 & 0.74 & 0.75 & 0.76 & 0.75 & 0.76 & $\mathbf{0 . 7 7}$ \\
\hline heart-statlog & 0.79 & 0.76 & $\mathbf{0 . 8 3}$ & 0.80 & 0.82 & 0.83 & 0.80 & 0.81 & 0.82 \\
\hline ionosphere & 0.90 & 0.90 & 0.94 & 0.92 & 0.91 & 0.94 & 0.94 & 0.93 & $\mathbf{0 . 9 5}$ \\
\hline messidor_features & 0.65 & 0.64 & 0.70 & 0.67 & 0.67 & 0.69 & 0.70 & 0.73 & $\mathbf{0 . 7 4}$ \\
\hline wifi & 0.97 & 0.97 & 0.98 & 0.98 & 0.97 & 0.98 & 0.98 & 0.98 & $\mathbf{0 . 9 9}$ \\
\hline
\end{tabular}


TABLE V

WEIGHTED RECALL VALUES FOR UCI DATASETS

\begin{tabular}{|l|c|c|c|c|c|c|c|c|c|}
\cline { 2 - 11 } \multicolumn{1}{c|}{} & \multicolumn{4}{c|}{ Base Learner } & \multicolumn{4}{c|}{$\begin{array}{c}\text { Meta Learner } \\
\text { Bagging }\end{array}$} & \multicolumn{3}{c|}{$\begin{array}{c}\text { Meta Learner } \\
\text { Rotation Forest }\end{array}$} \\
\hline Datasets & J48 & REPTree & Random Forest & J48 & REPTree & Random Forest & J48 & REPTree & Random Forest \\
\hline diabetes & 0.72 & 0.74 & 0.76 & 0.74 & 0.76 & 0.76 & 0.76 & 0.77 & $\mathbf{0 . 7 7}$ \\
\hline heart-statlog & 0.79 & 0.76 & $\mathbf{0 . 8 3}$ & 0.80 & 0.81 & 0.83 & 0.80 & 0.81 & 0.82 \\
\hline ionosphere & 0.90 & 0.90 & 0.94 & 0.92 & 0.91 & 0.94 & 0.93 & 0.93 & $\mathbf{0 . 9 5}$ \\
\hline messidor_features & 0.65 & 0.63 & 0.69 & 0.66 & 0.67 & 0.68 & 0.69 & 0.73 & $\mathbf{0 . 7 4}$ \\
\hline wifi & 0.97 & 0.97 & 0.98 & 0.98 & 0.97 & 0.98 & 0.98 & 0.98 & $\mathbf{0 . 9 9}$ \\
\hline
\end{tabular}

TABLE VI

WEIGHTED F-MEASURE VALUES FOR UCI DATASETS

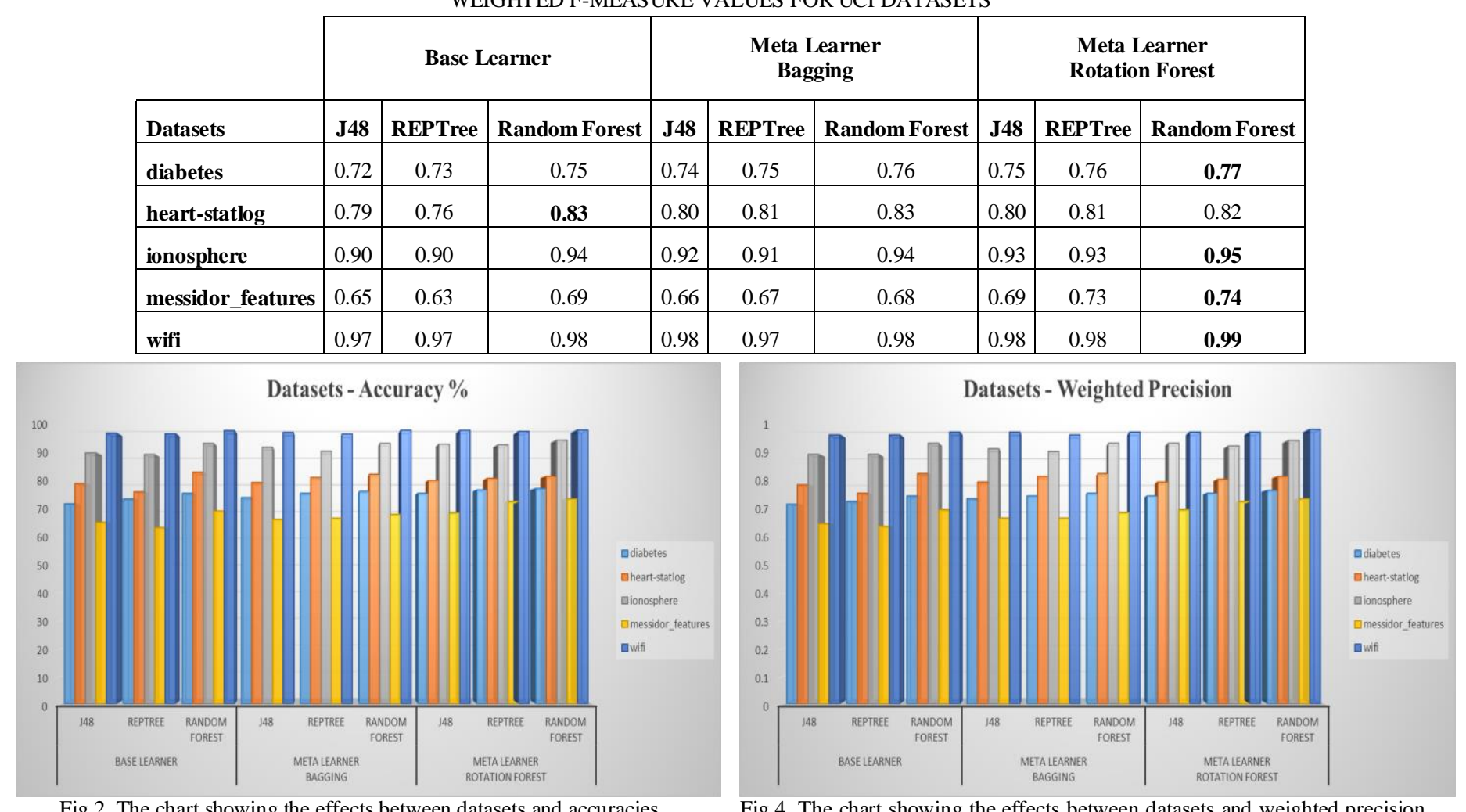

Fig.2. The chart showing the effects between datasets and accuracies

Fig.4. The chart showing the effects between datasets and weighted precision values

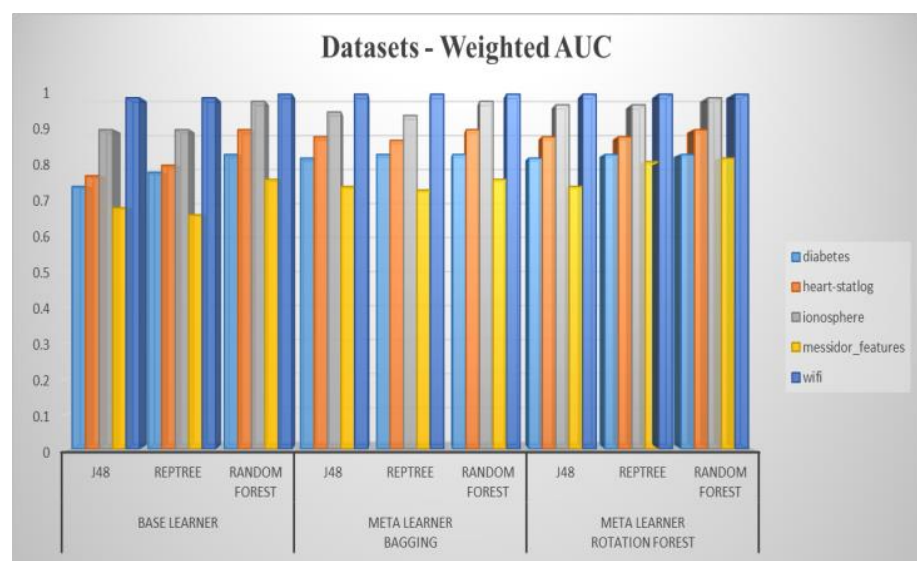

Fig.3. The chart showing the effects between datasets and weighted AUC values

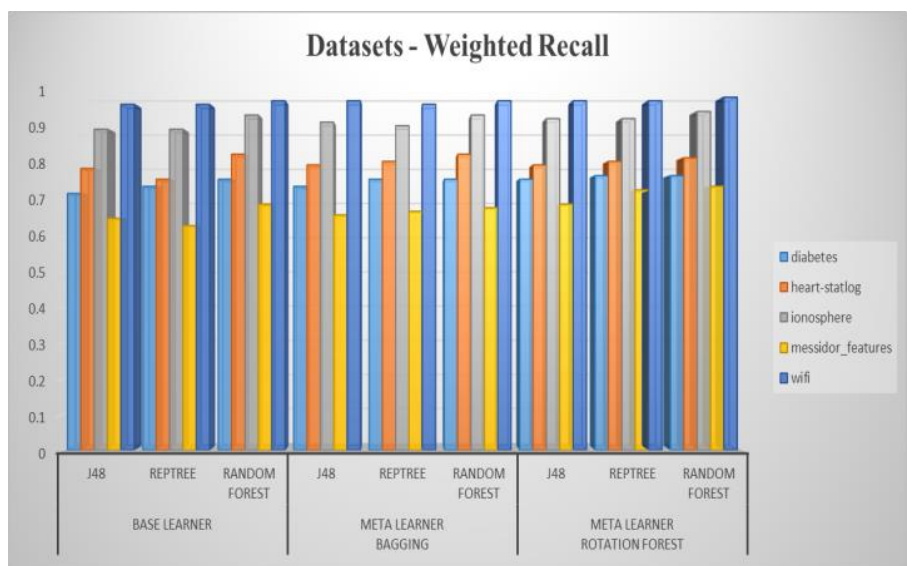

Fig.5. The chart showing the effects between datasets and weighted recall values 


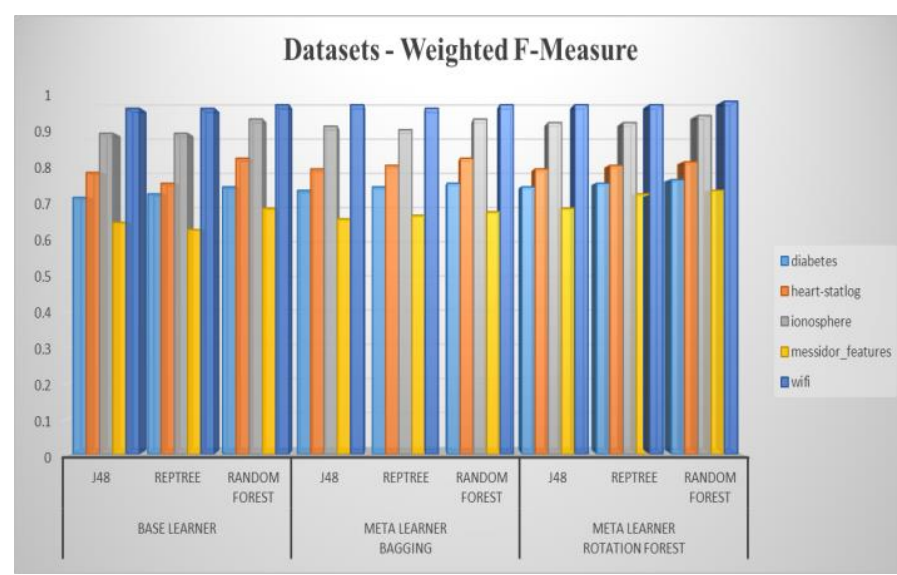

Fig.6. The chart showing the effects between datasets and weighted F-measure values

\section{CONCLUSION AND FUTURE WORK}

Nowadays, the use of the ensemble classifier is becoming more common due to its effectiveness and high performance for various fields such as machine learning and pattern recognition. In this study, a meta-ensemble learning method (Random Rotation Forest) based on Random Forest and Rotation Forest is proposed. Despite the fact that Random Rotation Forest can take more space and time for computations, this method yields more efficient results by using hybrid advantages of both algorithms.

As a future work, other hybridization of ensemble learning methods can be obtained. In addition, classifier weighting for base and meta-learners can be provided for improving classification. Feature weighting approaches can also be tried with diverse optimization algorithms [27].

\section{REFERENCES}

[1] T.G. Dietterich, Ensemble methods in machine learning, In International workshop on multiple classifier systems, Springer, Berlin, Heidelberg, 2000, pp. 1-15.

[2] W. Feng, W. Bao, Weight-Based Rotation Forest for Hyperspectral Image Classification, IEEE Geoscience and Remote Sensing Letters, 14(11), 2017, pp. 2167-2171.

[3] E. Aličković, A. Subasi, Breast cancer diagnosis using GA feature selection and Rotation Forest, Neural Computing and Applications, 28(4), 2017, pp. 753-763.

[4] M. Pal, Random forest classifier for remote sensing classification, International Journal of Remote Sensing, 26(1), 2005, pp. 217-222.

[5] A. Onan, Sentiment Analysis on Twitter Based on Ensemble of Psychological and Linguistic Feature Sets, Balkan Journal of Electrical and Computer Engineering, 6(2), 2018, pp. 1-9.

[6] W. Y. Loh, Classification and regression trees, Wiley Interdisciplinary Reviews: Data Mining and Knowledge Discovery, 1(1), 2011, pp. 14-23.

[7] W.N.H.W. Mohamed, M.N.M. Salleh, A.H. Omar, A comparative study of reduced error pruning method in decision tree algorithms, In Control System, Computing and Engineering (ICCSCE), 2012 IEEE International Conference on, 2012, pp. 392-397.

[8] L. Breiman, Random forests, Machine learning, 45(1), 2001, pp. 5-32.

[9] J.J. Rodriguez, L.I., Kuncheva, C.J. Alonso, Rotation forest: A new classifier ensemble method, IEEE transactions on pattern analysis and machine intelligence, 28(10), 2006, pp. 1619-1630.

[10] Y. Freund, R.E. Schapire, Experiments with a new boosting algorithm, In Icml, 96, 1996, pp. 148-156.

[11] K.H. Liu, D.S. Huang, Cancer classification using rotation forest, Computers in biology and medicine, 38(5), 2008, pp. 601-610.

[12] C.X. Zhang, J.S. Zhang, RotBoost: A technique for combining Rotation Forest and AdaBoost. Pattern recognition letters, 29(10), 2008, pp. 1524-1536.
[13] A. Ozcift, A. Gulten, Classifier ensemble construction with rotation forest to improve medical diagnosis performance of machine learning algorithms, Computer methods and programs in biomedicine, 104(3), 2011, pp. 443-451.

[14] P. Du, A. Samat, B. Waske, S. Liu, Z. Li, Random forest and rotation forest for fully polarized SAR image classification using polarimetric and spatial features, ISPRS Journal of Photogrammetry and Remote Sensing, 105, 2015, pp. 38-53.

[15] F. Lv, M. Han, Hyperspectral image classification based on improved rotation forest algorithm, Sensors, 18(11), 2018, 3601.

[16] A. Bagnall, A. Bostrom, G. Cawley, M. Flynn, J. Large, J. Lines, Is rotation forest the best classifier for problems with continuous features?, 2018, arXiv preprint arXiv:1809.06705.

[17] B.K. Singh, K. Verma, A.S. Thoke, Investigations on impact of feature normalization techniques on classifier's performance in breast tumor classification, International Journal of Computer Applications, 116(19), 2015, pp. 11-15.

[18] C.L. Devasena, Comparative analysis of random forest, REP tree and J48 classifiers for credit risk prediction, In International Journal of Computer Applications (0975-8887), International Conference on Communication, Computing and Information Technology (ICCCMIT2014), 2014, pp. 30-36.

[19] P. Hamsagayathri, P. Sampath, Decision tree classifiers for classification of breast cancer, Int. J. Curr. Pharm. Res, 9(2), 2017, 31.

[20] G. Biau, Analysis of a random forests model, Journal of Machine Learning Research, 13(Apr), 2012, pp. 1063-1095.

[21] O. Sagi, L. Rokach, Ensemble learning: A survey, Wiley Interdisciplinary Reviews: Data Mining and Knowledge Discovery, 8(4), e1249, 2018, pp. 1-18.

[22] C. Zhang, Y. Ma, Ensemble machine learning: methods and applications, Springer Science \& Business Media, 2012.

[23] G. Louppe, Understanding random forests: From theory to practice, arXiv preprint arXiv:1407.7502, 2014.

[24] UCI Machine Learning Repository, 2018, https://archive.ics.uci.edu/ml/datasets.html

[25] T. Fawcett, An introduction to ROC analysis, Pattern recognition letters, 27(8), 2006, pp. 861-874.

[26] T. Fawcett, ROC graphs: Notes and practical considerations for researchers, Machine learning, 31(1), 2004, pp. 1-38.

[27] E. Taşc1, O. Gökalp, A. Uğur, Development of a novel feature weighting method using CMA-ES optimization, In 2018 26th Signal Processing and Communications Applications Conference (SIU), IEEE, 2018, pp. $1-4$.

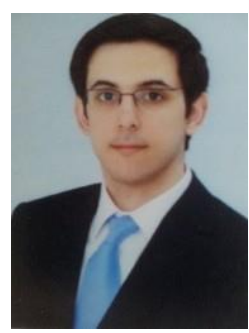

\section{BIOGRAPHIES}

ERDAL TAŞCI was born in 1989. He received the $\mathrm{BS}, \mathrm{MSc}$ and $\mathrm{PhD}$ degrees from the Ege University, Computer Engineering Department, Izmir, Turkey, in 2011, 2013 and 2018, respectively. He has been working as teaching assistant at the Department of Computer Engineering of Ege University, Izmir, Turkey. He has been reviewing for various journals including IEEE Transactions on Systems, Man, and Cybernetics: Systems, Computer Methods and Programs in Biomedicine and IET Image Processing. His research interests are pattern recognition, machine learning, image processing and data mining. 